\title{
Criminologie
}

\section{Le service de probation du Québec : évolution et rôles traditionnels}

\section{Paul-André Rivard}

Volume 12, numéro 2, 1979

Probation : aide ou contrainte?

URI : https://id.erudit.org/iderudit/017105ar

DOI : https://doi.org/10.7202/017105ar

Aller au sommaire du numéro

Éditeur(s)

Les Presses de l'Université de Montréal

ISSN

0316-0041 (imprimé)

1492-1367 (numérique)

Découvrir la revue

Citer cet article

Rivard, P.-A. (1979). Le service de probation du Québec : évolution et rôles traditionnels. Criminologie, 12(2), 7-23. https://doi.org/10.7202/017105ar d'utilisation que vous pouvez consulter en ligne.

https://apropos.erudit.org/fr/usagers/politique-dutilisation/ 


\section{LE SERVICE DE PROBATION DU QUÉBEC : EVOLUTION ET ROLES TRADITIONNELS}

Paul-André Rivard *

\section{LE SERVICE DE PROBATION DU QUEBEC : \\ SA CREATION ET SON EVOLUTIION}

En procédant à l'embauche et à l'assignation de ses premiers agents de probation, le ministère de la Justice du Québec jetait, en 1967, les bases de son service de Probation pour adultes. De ce fait, on pût dire que le service de Probation était créé et devenait partie intégrante du réseau des ressources mises à la disposition des tribunaux à juridiction criminelle et pénale au Québec.

La création du service de Probation répondait à un besoin pressant maintes fois exprimé par diverses personnes ou organismes impliqués, d'une façon ou d'une autre, dans le systeme judiciaire ou dans le système correctionnel.

A cette époque, plusieurs organismes d'enquête, dont le Comité canadien de la réforme pénale et correctionnelle, présidé par M. le juge Roger Ouimet, et la Commission d'enquête sur l'administration de la justice criminelle et pénale, présidée par M. le juge Yves Prévost, insistèrent sur l'importance de concrétiser une nouvelle philosophie pénale voulant que l'objectif fondamental de la justice criminelle soit d'assurer la protection de tous les membres de la société et, qu'à long terme, cette meilleure protection de la société réside dans la réhabilitation du délinquant. De semblables propos étaient tenus non seulement par les professionnels des sciences humaines et par des organismes sociaux ouvrant dans le domaine de la délinquance, mais aussi par des magistrats, tant individuellement que sur le plan collectif.

Il fallait donc intégrer cette philosophie nouvelle dans un système judiciaire jusque-là orienté vers le traitement punitif des délinquants et faire en sorte que l'on puisse davantage tenir

- Paul-Andre Rivard est directeur régional du service de Probation à Montréal et fut l'un des pionniers de ce service gouvernemental. Au cours de la preparation de cet article, il eft pour collaborateurs MM. Rene Bellerose, agent de probation à Trois-Riviéres, Jacques Boily, agent de probation $\mathbf{a}$ Sherbrooke et Robert Langlois, responsable du secteur ouest à Montréal. 
compte de l'homme criminel plutôt que de sa criminalité. Ainsi les efforts en vue de neutraliser sa déviance procéderaient dorénavant le plus souvent possible d'une lucide tentative de réinsertion sociale du contrevenant acceptant ainsi d'expérimenter de nouvelles avenues là où les méthodes plus traditionnelles avaient auparavant échoué.

En fait, par l'action d'agences sociales spécialisées æuvrant principalement dans les grands centres, le processus de changement était déjà amorcé. Plusieurs juges avaient eu l'occasion de se prévaloir de leurs services et s'étaient finalement engagés dans cette voie. On dénotait une plus grande ouverture d'esprit à l'égard du problème global de la délinquance et les agences en vinrent à ne plus pouvoir suffire à la tâche dans l'exercice d'un rôle relevant normalement de l'administration de la justice. Des représentations ont été faites de part et d'autre et c'est, en quelque sorte, l'action conjuguée de tous ces pionniers qui a suscité cette création tant souhaitée du service de Probation.

L'implantation de ce nouveau service au sein de l'appareil judiciaire ne s'est pas fait sans heurt et sans difficulté. De part et d'autre, juges, agents de probation, procureurs, policiers et justiciables ont tour à tour été frustrés dans leurs attentes. Non seulement chacun devait s'ajuster au changement, mais il fallait apprendre à se côtoyer, à cuvrer ensemble et comprendre que le même objectif général pourrait être poursuivi à partir de responsabilités, de points de vue et de rôles différents tout en acceptant que ces rôles puissent s'exercer de façon autonome.

Dans ce contexte, le service de Probation avait sârement un obstacle important à surmonter puisque étant en fait l'intrus ou, tout au moins, le nouveau venu dans le système, il se devait d'établir sa crédibilité, de démontrer que son apport aiderait a faire progresser l'administration de la justice. Ce cheminement du service de Probation était d'autant plus difficile à effectuer que ses agents, pour la plupart, ne possédaient que très peu d'expérience pratique.

En fait, même si le ministère de la Justice avait reconnu l'importance de rendre professionnel le rôle de l'agent de probation, il lui était pratiquement impossible de trouver, sur le marché du travail, un personnel qualifié et expérimenté, vraiment prêt, dans l'immédiat, à assumer pleinement un tel rôle. Conséquem- 
ment, ses efforts de recrutement se sont orientés vers les jeunes professionnels en sciences humaines récemment diplômés de nos universités. Au-delà de la bonne volonté et de l'engagement professionnel de ces derniers, il leur fallait faire le pont entre leurs connaissances académiques et la réalité pratique de la criminalité et du milieu judiciaire.

L'écart entre ces deux réalités ne pouvait être comblé que par l'acquisition de connaissances et d'expériences pratiques, tant sur le plan du comportement humain que sur celui du système judiciaire et par une aptitude à savoir proposer des approches ou théories nouvelles d'une façon compréhensible qui tienne compte du pouvoir et de la capacité d'évolution du système dans lequel ces agents travaillent.

La première phase de l'organisation du service de Probation visa à desservir le plus rapidement possible tous les districts judiciaires de la province en commençant, bien entendu, par les districts les plus importants en termes de besoins. Lorsque le service requis dans certains districts de moindre importance n'exigeait pas la dotation d'un agent permanent, cette responsabilité était confiée à un agent d'un bureau voisin.

Le district judiciaire de Montréal, par la densité de sa population représente plus de $40 \%$ de la clientèle judiciaire du Québec. Pour mieux répondre aux besoins de la cour et des justiciables, le territoire a été subdivisé en six zones d'activité où se sont implantées autant d'équipes de travail comprenant chacune de six à neuf agents de probation. Outre le fait, par ce rapprochement, de mieux desservir la clientèle spécifique de cette zone, une telle localisation dans un territoire donné permet aussi d'établir et de développer des relations étroites avec les ressources sociales de la communauté. Un autre avantage non négligeable de cette subdivision se retrouve dans la vie d'équipe des professionnels qui s'y trouvent. Il est plus facile d'y créer un esprit de collégialité et d'entraide que dans un groupe beaucoup plus étendu. Mentionnons que deux bureaux-satellites se greffent à la région de Montréal; il s'agit de Saint-Jérôme et de Joliette.

Sur le plan de la vie professionnelle il s'est établi un climat de recherche de l'excellence des services rendus. Le service 
de Probation a su proposer et faire accepter par la magistrature la priorité de la qualité du travail par rapport à la quantité. Ce qui est maintenant considéré comme un principe de fonctionnement du service constitue en fait la pierre angulaire qui guide constamment notre intervention. Ce principe amène nécessairement une plus grande sélection par les tribunaux de la clientèle qui nous est référée, mais nous considérons que les demandes qui nous sont adressées atteignent un fort taux de pertinence.

Cette recherche de l'excellence a amené le service de Probation à se doter de son propre service de formation et de perfectionnement qui a pour but d'améliorer le rendement professionnel. On a par ce moyen, organisé des programmes de perfectionnement destinés à compléter la formation et à diversifier les techniques d'intervention des agents. Dans ce cadre s'inscrivent des sessions de formation légale et celle concernant l'analyse transactionnelle, l'intervention à court terme, la psychosynthèse ou les toxicomanies. De plus, d'autres sessions s'adressent aux gestionnaires et peuvent même mener, selon les besoins et aptitudes, à des cours à plein temps en administration publique.

En somme, en dix ans d'existence, le service de Probation a réussi à s'implanter et à démontrer sa crédibilité.

De façon générale, la magistrature n'hésite pas à recourir aux services des agents de probation, tant pour des évaluations présentencielles que pour exercer la supervision en probation.

Sa structure administrative lui permet d'évoluer vers une qualité sans cesse accrue de son travail, grâce à la professionnalisation des agents, au dynamisme des relations de travail et aux efforts continus en matière de formation et de perfectionnement.

La situation actuelle du service au sein de l'appareil judiciaire lui permet d'entreprendre désormais des initiatives nouvelles ayant pour but l'implantation de programmes à vocation communautaire.

Il s'agit là tout aussi bien des travaux communautaires, des modalités de restitution, du dédommagement des victimes, 
moyens destinés à élargir l'éventail des mesures pénales alternatives à l'emprisonnement.

Parallèlement, le service de Probation assume maintenant des nouvelles fonctions connexes d'évaluation et de supervision, suite à la création de la Commission provinciale des libérations conditionnelles qui exerce sa juridiction sur tous les détenus des prisons provinciales.

En somme, le service de Probation est en plein essor et ses possibilités de développement sont à la mesure des besoins de la clientèle à desservir.

\section{LES RÓLES TRADITIONNELS}

La Loi de la probation et des établissements de détention adoptée par l'Asemblée nationale du Québec et sanctionnée le 27 mai $1969^{1}$, donnait, non seulement un cadre juridique au service de Probation, mais agissait en parallèle au Code criminel en créant la possibilité, pour les tribunaux, de recourir aux services de l'agent de probation à l'égard d'individus condamnés pour des infractions à des lois statutaires provinciales.

De plus, il est important de souligner l'obligation qu'elle crée, au directeur général du service et à l'agent de probation, non seulement de favoriser l'application des mesures de probation à l'égard de personnes reconnues coupables d'infraction au Code criminel ou à toute loi pénale, mais aussi de favoriser la réinsertion sociale des dites personnes. Cette ligne de conduite constitue, en fait, le fondement de l'intervention du service de Probation et de ses agents et oriente toutes ses interventions. C'est probablement la première fois au Canada que l'on retrouve, clairement exprimé, ce qui est habituellement sous-entendu dans d'autres lois.

Du Code criminel et de la loi provinciale sont issus les deux rôles majeurs qu'un agent de probation est appelé à assumer, soit la préparation de rapports présentenciels et la surveilance de justiciables durant les périodes probatoires. Ces deux sphères d'activité auxquelles viennent se greffer d'autres initiatives, ont évolué avec les années et nous croyons pertinent aujourd'hui d'en faire la présentation.

1. Statuts du Québec, 1969, chapitre 21. 


\section{A. Le rapport présentenciel}

Avant de développer toutes les facettes qui concernent l'Evaluation présentencielle et le rapport qui en fait foi, il convient de situer globalement le lecteur par une définition générale indiquant sommairement les dimensions du document connu sous le nom de * rapport présentenciel . Les textes de loi étant muets à ce sujet, le service de Probation a développé peu à peu une définition qui lui est personnelle et qui, semble-t-il, respecte la réalité.

Le rapport présentenciel est un document écrit préparé à la demande de la cour, au sujet d'une personne reconnue coupable, et destiné à aider le juge à rendre sentence en fournissant un portrait social de ce justiciable, un diagnostic sur sa dangerosité et un pronostic sur sa capacité de rêinsertion sociale.

Parmi tant d'autres, cette définition a pour avantage certain de regrouper, sous un même titre, tous les éléments essentiels du rapport présentenciel tel qu'il est voulu par la loi ainsi que par la coutume qui s'établit localement.

Les origines légales du rapport présentenciel proviennent de deux sources différentes :

a) de source fédérale, qui a juridiction exclusive en matière de législation et de poursuite criminelle;

b) de source fédérale ou provinciale, selon le cas, en matière pénale mais non criminelle.

La loi fédérale contenue dans le Code criminel stipule ce qui suit :

662(1) Lorsqu'un accusé autre qu'une corporation plaide coupable ou est reconnu coupable d'une infraction, un agent de probation doit, s'il est requis de le faire par une cour, préparer et déposer à la Cour un rapport écrit concernant l'accusé, afin d'aider celle-ci à imposer une sentence ou à décider si l'accusé devrait être libéré en application de l'article 662.I.

(2) Lorsqu'un rapport est déposé à la Cour en vertu du paragraphe (1), le greffier de la Cour doit immédiatement faire fournir une copie du rapport à l'accusé ou à son procureur ainsi qu'au poursuivant.

D'autre part, les dispositions régissant le rapport présentenciel en matière provinciale, se retrouvent dans la Loi de la 
probation et des établissements de détention, qui stipule à l'article 12 :

Tout agent de probation doit :

a) faire enquête, à la demande du tribunal qui déclare une personne coupable, sur ses antécédents, son caractère et la possibilité de sa réhabilitation ainsi que sur tout autre sujet qui la concerne et que lui indique le tribunal ;

b) faire au tribunal un rapport écrit de son enquête pour l'aider à imposer une sentence au contrevenant;

c) conseiller le tribunal, à sa demande, sur les conditions qui devraient être prescrites par toute ordonnance de libération conditionnelle ou surveillée.

Nonobstant ces deux sources légales, l'expérience vécue par le service de Probation du Québec, outre quelques rares cas d'exception, démontre que le rapport présentenciel est presque exclusivement produit aux termes de procédures criminelles relevant de la loi fédérale et que bien peu de tribunaux se servent de la loi provinciale pour des contrevenants à des statuts provinciaux. Cette constatation nous force donc à traiter du rapport présentenciel sous un seul angle.

\section{La dimension légale}

L'agent de probation doit, lorsque requis par la cour, préparer et produire un rapport concernant le justiciable devant recevoir une sentence. De ce fait, il devient un mandataire de la justice. Il doit la vérité à la juridiction qui l'a mandaté et avec laquelle il a son seul lien. Son objectivité et son impartialité doivent s'identifier à celle de la cour car sa crédibilité en dépend.

L'introduction du rapport présertenciel dans le processus judiciaire doit respecter les règles fondamentales de notre droit qui exige que tous les éléments admis en preuve soient portés à la connaissance des parties en cause. Conséquemment, le rapport doit non seulement être écrit, mais des copies doivent être remises à l'accusé ou à son procureur ainsì qu'au représentant de la poursuite. A ce sujet et en vue de permettre la consultation du rapport avant le jour de l'audience, le service de Probation se charge généralement de faire parvenir les copies du rapport aux procureurs de la défense, de même que la poursuite. Une telle pratique tend à accélérer le processus judiciaire. 
Considérant qu'une corporation puisse être accusée d'infractions ou d'actes criminels, le législateur a jugé utile de préciser que la possibilité de recourir au rapport présentenciel ne pouvait s'appliquer qu'à des personnes.

Notre système judiciaire, d'inspiration britannique, divise le procès pénal en deux phases distinctes, celle du jugement, par laquelle est déterminée la culpabilité ou l'innocence de la personne et celle de la sentence, lorsque cette dernière a été déclarée coupable. Le processus d'évaluation et de rapport présentenciel ne peut donc se situer que dans cette seconde phase.

Même si le Code criminel est pratiquement muet quant au contenu du rapport présentenciel, la pratique et la jurisprudence indiquent clairement qu'il s'agit d'une évaluation sociale. II faut savoir présenter à la cour le portrait social le plus fidèle possible de l'accusé, dégager le potentiel de dangerosité qu'il peut représenter pour la société et évaluer son aptitude à en réintégrer les rangs. Outre ce portait général du contrevenant, il est aussi important de bien percevoir les besoins de la cour lorsqu'elle s'adresse à l'agent de probation pour obtenir un rapport. En fait, quelle que soit la valeur intrinsèque du document présenté, il perd énormément de son utilité s'il ne sait pas répondre adéquatement aux attentes du requérant.

La crédibilité d'un rapport implique qu'il possède des qualités fondamentales certaines qui sont : l'exactitude, la pertinence, l'objectivité, la cohérence, la précision, la concision et, comme il va de soi, la forme soignée.

\section{L'évaluation sociale}

Afin de produire un rapport qui puisse permettre d'atteindre l'objectif poursuivi conformément aux critères de qualité énumérés précédemment, l'agent de probation procède à une évaluation au cours de laquelle il recueille toutes les données susceptibles de le guider et de l'éclairer dans l'accomplissement de sa tâche. Il serait onéreux et peu utile de reprendre ici le processus bien connu de l'enquête sociale, mais il est bon de s'arrêter sur certains problèmes particuliers avec lesquels, dans les circonstances, l'agent de probation est habituellement confronté. 
On peut relever entre autres la confidentialité à laquelle sont normalement astreints les intervenants sociaux ainsi que la sélection à être consignée au rapport.

Il est d'importance capitale que le rôle de mandataire de la justice qu'exerce l'agent de probation soit perçu avec la plus grande exactitude possible, non seulement par le contrevenant sujet à l'évaluation, mais aussi par toutes les personnes-ressources qui acceptent d'y participer. Tous doivent percevoir clairement que l'information transmise à un agent de probation est susceptible d'être utilisée pour les fins du rapport à la cour et qu'il ne peut exister de confidentialité à ce niveau.. Personne, ni même l'accusé, n'étant légalement tenu de collaborer avec l'agent de probation à la préparation d'un rapport présentenciel, il importe que ceux et celles qui sont approchés puissent faire un choix en toute connaissance de cause et qu'ils ne soient jamais placés en situation de prétendre que leur confiance a été trahie, que des informations données sous le sceau de la confidentialité ont été divulguées.

Ce genre de mise en garde devient encore plus nécessaire lorsque l'agent transige avec des personnes mal informées, simples, sans instruction ou même déficientes. Certains pourront dire que cette façon de procéder constitue en fait une invitation à se taire, à ne rien dire qui puisse être compromettant ; cependant, ce souci d'intégrité, chez l'agent de probation, contribue à créer un climat de confiance, à le faire percevoir comme étant capable de droiture et lui assure généralement une collaboration accentuée de la part de ceux auxquels il fait appel.

De toute façon il vaut mieux risquer de perdre quelques informations, plutôt que d'entacher sa crédibilité.

Absence de confidentialité ne veut pas dire licence quant à la sélection et à la transmission de l'information. C'est ainsi que, conscient de ses responsabilités, l'agent de probation fera montre de la plus grande discrétion possible tout en respectant son mandat à l'égard du tribunal. Ses relations avec les justiciables et leurs proches l'amèneront sûrement à recevoir une information qui, dans un autre contexte, eût été qualifiée de confidentielle. En outre, beaucoup d'autres informations de connaissance générale ont pu lui être transmises sans pour autant que ces informations, même si elles ont été utiles pour une 
meilleure compréhension d'un cas, aient une pertinence quelconque avec le besoin manifesté par la cour.

La sélection des informations à être transmises à la cour fait donc largement appel au jugement et à la responsabilité professionnelle de l'agent de probation. Ce dernier doit apprécier les informations qu'il possède et évaluer la pertinence de les inclure dans un rapport. A cet égard, on peut citer, par exemple, les déclarations d'une épouse qui risquerait ultérieurement de subir les sévices de son mari parce qu'elle aurait divulgué certaines informations sur son comportement, les confidences de personnes qui vivent et qui continueront à vivre avec l'accusé et, de façon générale, toutes les informations que des témoins, par crainte, pudeur ou pour d'autres raisons, se refuseraient à venir supporter devant le tribunal.

\section{Le rapport}

Au-delà de son obligation de transmettre à la cour toute l'information pertinente concernant un accusé, l'agent de probation doit se prononcer sur les risques encourus par la société dû à la délinquance du justiciable et sur sa capacité de réinsertion sociale. Cette particularité, non spécifiquement requise par la loi, mais voulue par le service de Probation, dans l'optique du caractère professionnel du rôle de l'agent de probation, est généralement bien acceptée par les juges, même si elle constitue une expression d'opinion de la part de l'agent de probation.

Cette façon de voir et d'agir, ces expressions d'opinion, ont bien sûr créé des remous et provoqué des réactions pas toujours favorables. Cependant on peut dire que ces réactions se sont généralement rapportées à la forme plutôt qu'au fond de cette intervention. C'est ainsi que même si des difficultés ont été rencontrées et se rencontrent encore à l'occasion, on constate qu'elles s'amenuisent et tendent à disparaître dans la mesure où le champ d'intervention de l'agent de probation se précise, dans la mesure où il sait reconnaître les limites de son rôle et dans la mesure aussi où il s'applique à bien percevoir les rôles des divers autres intervenants impliqués dans le processus judiciaire et à les repecter.

Ce mode d'intervention de l'agent de probation s'étant révélé utile et efficace, il y a lieu de laisser l'expérience le mo- 
deler, le raffiner. Devant les efforts déployés en vue d'humaniser la justice, en vue de tenir compte de la réalité sociale dans laquelle évoluent les justiciables, le rapport présentenciel devient un instrument privilégié. Au-delà des erreurs de parcours qui peuvent se commettre, des corrections et ajustements nécessaires, il demeure que l'on doive veiller à ce qu'il conserve et améliore sa qualité professionnelle et qu'il demeure un appui certain pour le juge qui s'en prévaut.

\section{Historique et perspective d'avenir}

Après avoir défini le rapport présentenciel, tant dans sa réalité pratique que dans son contexte légal, il est intéressant de voir comment ce moyen d'information a été utilisé par les tribunaux depuis qu'il relève du service de Probation.

Une première réponse peut être fournie par l'analyse des statistiques qui démontrent la progression de son utilisation. En se référant, à cet égard, aux données publiées dans les rapports annuels de la direction générale de la Probation et des établissements de détention, le service de Probation a produit en 1977 : 2978 rapports présentenciels comparativement à 427 en 1968.

On constate aisément qu'au cours de ces dix années le rapport présentenciel a pris sa place dans le processus judiciaire et son utilisation par les tribunaux est devenue de plus en plus fréquente. Conséquemment, on peut en déduire que le taux de crédibilité des agents de probation a dû évoluer en parallèle.

Ces statistiques des 10 dernières années démontrent en outre une évolution de l'utilisation du rapport présentenciel à l'égard d'une criminalité de plus en plus sérieuse.

Alors qu'au début du service, une grande partie des rapports était préparée en regard d'individus ayant commis des infractions mineures, on peut dire qu'en 1977, au-delà de la moitié des rapports présentenciels concernait des justiciables ayant commis des infractions majeures, telles que vols qualifiés, extorsion, viol, assauts contre la personne, trafic de stupéfiants, vol par effraction, etc. Encore là nous pouvons y voir un indice de la confiance accordée par les tribunaux aux agents de probation et à leurs rapports présentenciels, ainsi que le souci de se pencher sur le problème social de l'individu, même lorsque, 
dans plusieurs cas, l'emprisonnement éventuel est pratiquement inévitable.

Que réserve l'avenir au rapport présentenciel ?

Dans sa forme et sa structure actuelle, il ne fait aucun doute qu'il répond à un besoin et il est souhaitable de le voir progresser vers une qualité sans cesse accrue. Cependant, le service de Probation reconnaît que l'examen approfondi de chacun des cas n'est pas toujours nécessaire et que dans certains, des études plus sommaires, plus expéditives, pourraient satisfaire aux besoins de la cour. C'est pourquoi on étudie présentement les résultats d'expériences faites par d'autres services, notamment en ce qui concerne les enquêtes et rapports sommaires.

Dans cette optique de la recherche de nouvelles avenues, a été instauré, à Montréal, un rôle d'agent de liaison auprès des tribunaux. En plus de minimiser les problèmes d'ordre technique lors de dénonciations ou de modifications des conditions de probation, l'agent de liaison s'emploie à percevoir les besoins des tribunaux en rapport avec chacune des demandes d'évaluation et à procéder, s'il y a lieu, à des études sommaires de cas. Cette nouvelle approche est très appréciée des juges et facilite grandement le dialogue et les échanges avec le service de Probation.

Malgré qu'il soit souhaitable d'être inventifs, de diversifier notre approche quant au rapport présentenciel et de rejoindre une plus grande clientèle, il faut savoir maintenir notre objectif premier de la qualité des services. Il faut aussi demeurer à l'écoute des tribunaux, être attentifs à leurs besoins et savoir leur proposer des méthodes d'intervention qui y répondent.

\section{B. La probation}

\section{La probation au Canada et au Québec}

La probation a trouvé ses premières applications dans ce qu'on pouvait alors appeler : \& La Loi pour permettre la libération conditionnelle des délinquants à leur premier délit s. Cette loi existait déjà en 1889, mais non conciliée dans un même texte. Il s'agissait d'une modalité applicable sous certaines conditions et pour certains délinquants à leur premier délit. Cependant l'infraction en question ne devait pas être passible d'un 
emprisonnement de plus de deux ans. Il s'agissait en somme d'une disposition judiciaire à certaines conditions, mais sans surveillance, ni limite de temps.

Progressivement, la loi a été amendée favorisant l'application de la probation. Le Code criminel canadien d'alors (1900) prévoyait une telle disposition judiciaire avec amendement au chapitre des antécédents judiciaires. De ce fait, la restriction d'application (premier délit) se trouvait écartée. Extension également au chapitre de la sanction à savoir qu'elle pouvait même s'appliquer aux délits punissables par plus de deux ans d'emprisonnement. Toutefois le consentement du procureur de la Couronne était requis dans certains cas.

En 1921, le Parlement canadien ajoutait à la probation, la notion de surveillance par une personne désignée par la cour. Cette notion de surveillance allait inciter les provinces à légiférer pour nommer des adjoints à la cour.

La modification du Code criminel en 1955 précisait que la probation ne pouvait s'échelonner sur plus de deux ans. On parlait alors plutôt d'une sentence suspendue applicable à des individus ayant commis leur premier délit ou à des récidivistes ne possédant pas plus d'un antécédent judiciaire. En outre, si cet antécédent judiciaire était de même nature que la récidive, il devait s'écouler une période de cinq années entre la commission de ces deux délits. Une personne désignée par la cour pouvait assumer la surveillance du délinquant et devait faire rapport au tribunal en cas de non-respect des conditions émises.

Depuis les dispositions du Code ont été modifiées et élargies au point où il ne subsiste pratiquement plus de limites à l'émission d'une ordonnance de probation. Seules certaines infractions pour lesquelles il existe une peine minimale d'emprisonnement ne permettent pas l'utilisation du sursis de sentence avec probation. Même les antécédents judiciaires ne constituent plus un empêchement à l'émission d'une telle ordonnance.

Il est bon de rappeler ici que la principale juridiction exercée en matière de probation est de source fédérale. Même si la Loi de la probation et des établissements de détention (art. 5) permet l'octroi d'ordonnance de probation à l'égard de contrevenants ayant commis des infractions à des statuts provinciaux, il faut admettre qu'à la connaissance du service de Probation, 
une telle disposition n'a jamais été utilisée par les tribunaux, de sorte que l'expérience vécue se situe en totalité au niveau des actes et infractions criminelles de juridiction fédérale.

La loi fédérale désigne maintenant sous le nom d'agent de probation les personnes qui assument la surveillance des contrevenants soumis à un tel type d'ordonnance. Les textes de loi traitent désormais \& d'ordonnance de probation par laquelle un inculpé peut recouvrer sa liberté moyennant le respect des conditions émises par la cour. La durée d'une ordonnance pour un délit criminel ne peut s'échelonner sur plus de trois ans pour une infraction aux lois fédérales et sur plus de deux ans pour une infraction aux statuts provinciaux.

On doit néanmoins faire cette nuance à l'effet qu'une ordonnance de probation n'implique pas automatiquement l'intervention d'un agent de probation. Cette intervention ne survient que si la cour le spécifie comme une modalité de l'ordonnance. Sont par contre inhérentes à l'ordonnance de probation, les trois conditions suivantes :

- Ne pas troubler l'ordre pubic;

- Avoir une bonne conduite ;

- Comparaître devant la cour lorsque requis.

Il est essentiel qu'il y ait reconnaissance de culpabilité de l'accusé avant que le juge ne puisse imposer une ordonnance de probation; l'ordonnance de probation telle que stipulée par le Code ne s'adresse qu'aux individus adultes ou à l'exception à des délinquants mineurs déférés par le tribunal de la jeunesse. L'âge, la réputation de l'accusé, la nature de l'infraction, les circonstances entourant le délit peuvent guider le juge dans son choix de recourir ou non à cette disposition judiciaire.

En somme, le rappel de l'évolution légale de la probation dans notre pays, permet de comprendre qu'il existe depuis nombre d'années dans notre système judiciaire une loi permettant au juge de recourir à une disposition autre que l'amende ou la détention lorsqu'il doit rendre une sentence à l'égard d'un délit commis.

Si, au Québec, le service de Probation n'existe que depuis 1967, il n'en demeure pas moins que nos cours de justice ont mis à profit leurs prérogatives en matière de probation long- 
temps avant cette date. On peut rappeler notamment que des travailleurs sociaux cuvrant dans des agences sociales répondaient aux demandes des cours pour préparer les rapports avant sentence et assumer la surveillance des justiciables d'alors. Il faut dire aussi qu'à défaut d'agents de probation, les juges imposaient depuis longtemps des sursis de sentence sans surveillance, faisant ainsi une confiance totale aux sursitaires.

\section{Réalité pratique de la probation au Québec}

Il serait prétentieux de prétendre que la probation au Québec diffère grandement de ce qui se fait ailleurs en la matière.

Disons d'abord que dans les premières années de son existence, le service de Probation s'est attaché particulièrement à la préparation du rapport présentenciel et, sans pour autant vouloir négliger la probation, il faut admettre que cette dernière est passée quelque peu au second rang dans les étapes d'implantation. Il faut admettre en outre, que le personnel professionnel manquait nettement d'expérience et éprouvait beaucoup de difficultés à saisir le sens profond de cette forme d'intervention, à conjuguer contrôle, autorité et assistance sociale.

Tous ces problèmes se sont graduellement résorbés. Le personnel a acquis maturité et expérience et le service de Probation a précisé ses attentes par un guide et des directives concernant l'application de cette mesure judiciaire et sociale.

Là où le service de Probation tend à se distinguer, c'est lorsqu'il cherche à limiter le nombre de surveillés en recrutant ces derniers chez ceux qui se montrent ouverts à une intervention sociale et désireux de bénéficier de l'assistance des agents de probation. Il faut admettre de façon générale que la clientèle d'un service de probation n'est pas une clientèle volontaire et que ses contacts avec l'agent sont rendus plus difficiles à cause, principalement, de l'obligation de contrôle imposée à ce dernier.

Sans d'aucune façon négliger l'aspect contrôle de son rôle, le service de Probation recherche particulièrement le changement chez sa clientèle. $\AA$ cette fin, il offre des services et se rend disponible à l'égard de ceux qui veulent bien en profiter. Il accorde une attention spéciale à la motivation des contrevenants 
à effectuer les ajustements nécessaires dans leur vie. C'est pourquoi, d'une part, il essaiera, par les moyens légaux, de s'affranchir de l'obligation de contrôle d'individus qui n'ont pas besoin ou ne veulent pas de ses services et, d'autre part, il évitera de confier à ses agents une charge de travail exagérée qui aurait pour effet de faire disparaître toute espèce de disponibilité à l'égard des demandes des justiciables qui lui sont confiés.

Étant ainsi centré sur la croissance personnelle et sur le comportement social du contrevenant, l'agent de probation est amené à intervenir, tantôt en conseiller budgétaire, tantôt comme conseiller à l'emploi ou encore comme intermédiaire dans un conflit familial ou conjugal. Dans certains cas, par sa compétence dans un type particulier d'intervention, il s'emploiera à guider le client vers la solution de ses problèmes ou la réponse à ses besoins; en d'autres occasions, il agira comme intermédiaire pour référer le cas à une ressource communautaire spécialisée. D'une manière générale, préalablement à une connaissance professionnelle, l'agent de probation doit être authentique face aux valeurs qu'il propose, franc et sincère dans ses relations avec le justiciable qu'il ne doit pas juger. Ce dernier se servira selon sa motivation, de l'agent de probation comme d'un tremplin permettant une croissance personnelle.

\section{Utilisation de la probation}

Au terme de l'année 1977, le service de Probation comptait près de 3000 cas actifs en surveillance. Selon le dernier rapport annuel, on constate une augmentation d'environ $11 \%$.

Depuis la création du service, le nombre de contrevenants qui lui sont confiés s'est accru en proportion des effectifs alloués. Les tribunaux, conscients des limites de ces effectifs et ayant admis la priorité de la qualité de l'intervention sur la quantité de dossiers traités, se considèrent quelque peu liés par ce principe et évitent ainsi de surcharger inutilement les agents de probation.

Selon les statistiques, il semble que les ordonnances de probation soient davantage accordées pour les délits de type vol par effraction (19\%) et vol de plus de $\$ 200$ (10\%). Par ailleurs, les délits tels que le vol qualifié et les crimes sexuels comptent pour $13 \%$ des cas référés au service. 


\section{Perspectives d'avenir}

Que se passera-t-il à court et à long termes en probation ?

Après avoir traversé sa phase d'implantation, le service de Probation devra s'appliquer à brève échéance à analyser les résultats de son intervention.

Il devra aussi par un suivi constant des dossiers et par une intervention efficace pouvoir réduire de façon appréciable la durée de l'intervention des agents. De plus, l'analyse des cas faisant habituellement l'objet d'un sursis sans surveillance pourrait lui permettre de déceler une clientèle que les tribunaux auraient intérêt à lui confier.

Somme toute, il ne faut pas craindre de ré-évaluer toute notre action, à ce niveau, d'en mesurer l'efficacité et de savoir la réajuster selon les besoins. 